\title{
A radial estimate for the maximal operator associated with the free Schrödinger equation
}

\author{
by \\ Sichun WANG (Ottawa)
}

Dedicated to the fond memories of Wang Fang Mei and Wang Jiao Yun

Abstract. Let $d>0$ be a positive real number and $n \geq 1$ a positive integer and define the operator $S_{d}$ and its associated global maximal operator $S_{d}^{* *}$ by

$$
\begin{aligned}
& \left(S_{d} f\right)(x, t)=\frac{1}{(2 \pi)^{n}} \int_{\mathbb{R}^{n}} e^{i x \cdot \xi} e^{i t|\xi|^{d}} \widehat{f}(\xi) d \xi, \quad f \in \mathcal{S}\left(\mathbb{R}^{n}\right), x \in \mathbb{R}^{n}, t \in \mathbb{R}, \\
& \left(S_{d}^{* *} f\right)(x)=\sup _{t \in \mathbb{R}}\left|\frac{1}{(2 \pi)^{n}} \int_{\mathbb{R}^{n}} e^{i x \cdot \xi} e^{i t|\xi|^{d}} \widehat{f}(\xi) d \xi\right|, \quad f \in \mathcal{S}\left(\mathbb{R}^{n}\right), x \in \mathbb{R}^{n},
\end{aligned}
$$

where $\widehat{f}$ is the Fourier transform of $f$ and $\mathcal{S}\left(\mathbb{R}^{n}\right)$ is the Schwartz class of rapidly decreasing functions. If $d=2, S_{d} f$ is the solution to the initial value problem for the free Schrödinger equation (cf. (1.3) in this paper). We prove that for radial functions $f \in \mathcal{S}\left(\mathbb{R}^{n}\right)$, if $n \geq 3$, $0<d \leq 2$, and $p \geq 2 n /(n-2)$, the maximal function estimate

$$
\left(\int_{\mathbb{R}^{n}}\left|\left(S_{d}^{* *} f\right)(x)\right|^{p} d x\right)^{1 / p} \leq C\|f\|_{H_{s}\left(\mathbb{R}^{n}\right)}
$$

holds for $s>n(1 / 2-1 / p)$ and fails for $s<n(1 / 2-1 / p)$, where $H_{s}\left(\mathbb{R}^{n}\right)$ is the $L^{2}$-Sobolev space with norm

$$
\|f\|_{H_{s}\left(\mathbb{R}^{n}\right)}=\left(\int_{\mathbb{R}^{n}}\left(1+|\xi|^{2}\right)^{s}|\widehat{f}(\xi)|^{2} d \xi\right)^{1 / 2} .
$$

We also prove that for radial functions $f \in \mathcal{S}\left(\mathbb{R}^{n}\right)$, if $n \geq 3, n /(n-1)<d<n^{2} / 2(n-1)$, then the estimate

$$
\left(\int_{\mathbb{R}^{n}}\left|\left(S_{d}^{* *} f\right)(x)\right|^{2 n /(n-d)} d x\right)^{(n-d) / 2 n} \leq C\|f\|_{H_{s}\left(\mathbb{R}^{n}\right)}
$$

holds for $s>d / 2$ and fails for $s<d / 2$. These results complement other estimates obtained by Heinig and Wang [7], Kenig, Ponce and Vega [8], Sjölin [9]-[13], Vega [19]-[20], Walther [21]-[23] and Wang [24].

2000 Mathematics Subject Classification: Primary 42B25; Secondary 42A45.

Key words and phrases: free Schrödinger equation, maximal functions, spherical harmonics, oscillatory integrals. 
1. Introduction. Let $P$ be a real-valued smooth phase function and $S_{P}$ the integral operator

$$
\left(S_{P} f\right)(x, t)=(2 \pi)^{-n} \int_{\mathbb{R}^{n}} e^{i x \cdot \xi} e^{i t P(\xi)} \widehat{f}(\xi) d \xi, \quad x \in \mathbb{R}^{n}, t \in \mathbb{R},
$$

where $\widehat{f}$ is the Fourier transform of $f$ defined by

$$
\widehat{f}(\xi)=\int_{\mathbb{R}^{n}} e^{-i \xi \cdot x} f(x) d x .
$$

Then $u(x, t)=\left(S_{P} f\right)(x, t)$ is the formal solution of the dispersive initial value problem

$$
\begin{aligned}
\frac{\partial u}{\partial t}(x, t) & =i P(D) u(x, t), \quad x \in \mathbb{R}^{n}, t \in \mathbb{R}, \\
u(x, 0) & =f(x),
\end{aligned}
$$

where $D=-i\left(\partial / \partial x_{1}, \ldots, \partial / \partial x_{n}\right)$, and $P(D)$ is defined by

$$
P(D) u(x)=(2 \pi)^{-n} \int_{\mathbb{R}^{n}} e^{i x \cdot \xi} P(\xi) \widehat{u}(\xi) d \xi .
$$

If $P(\xi)=|\xi|^{2},(1.2)$ reduces to the initial value problem for the free Schrödinger equation

$$
\frac{\partial u}{\partial t}(x, t)=-i \Delta_{x} u(x, t), \quad u(x, 0)=f(x),
$$

while the case $P(\xi)=\xi^{3}$, with $n=1$, yields the linear version of the Korteweg-de Vries equation. For other examples of phase functions, see [7]-[8].

In this paper the phase function $P$ is assumed to be radial and realvalued, that is, $P(\xi)=\varphi(|\xi|), \xi \in \mathbb{R}^{n}$, where $\varphi \in C^{1}(0, \infty)$ is real-valued, for example $P(\xi)=|\xi|^{d}, d>0$. In this latter case the corresponding integral operator (1.1) is denoted by $\left(S_{d} f\right)(x, t)$. If $f \in \mathcal{S}\left(\mathbb{R}^{n}\right)$, the Schwartz class of rapidly decreasing functions, an application of Parseval's formula shows that $\left(S_{d} f\right)(x, t)=(2 \pi)^{-n}\left(K_{t}^{d} * f\right)(x)$, where $K_{t}^{d}(x)=\int_{\mathbb{R}^{n}} e^{-i x \cdot \xi} e^{i t|\xi|^{d}} d \xi$. In particular, $u(x, t)=(2 \pi)^{-n}\left(K_{t}^{2} * f\right)(x)$ is the formal solution of the Schrödinger equation (1.3).

In 1979, Carleson [3] posed the following problem: Find the smallest $s>0$ such that for any compactly supported $f \in H_{s}\left(\mathbb{R}^{n}\right)$, the limit $\lim _{t \rightarrow 0^{+}}(2 \pi)^{-n}\left(K_{t}^{d} * f\right)(x)=f(x)$ exists a.e. Here $H_{s}\left(\mathbb{R}^{n}\right)$ are the $L^{2}$-Sobolev spaces with norm

$$
\|f\|_{H_{s}}=\left(\int_{\mathbb{R}^{n}}\left(1+|\xi|^{2}\right)^{s}|\widehat{f}(\xi)|^{2} d \xi\right)^{1 / 2} .
$$

In one dimension this problem is completely solved in the sense that $(2 \pi)^{-1}\left(K_{t}^{d} * f\right)(x) \rightarrow f(x)$ a.e., as $t \rightarrow 0^{+}$, if $f \in H_{s}(\mathbb{R}), s \geq 1 / 4$, with 
$s=1 / 4$ best possible ([4]). However, if $n \geq 2$, Carleson's problem is still open, although many partial results are known (cf. [7]-[13], [18]-[24]).

The almost everywhere convergence of the solution to initial data $f$ is closely related to regularity properties of the local and global maximal operators

$$
\left(S_{d}^{*} f\right)(x)=\sup _{0<t<1}\left|\left(S_{d} f\right)(x, t)\right|, \quad\left(S_{d}^{* *} f\right)(x)=\sup _{t \in \mathbb{R}}\left|\left(S_{d} f\right)(x, t)\right| .
$$

In fact Sjölin [9] proved that if for any $T>0$, there is a constant $C>0$ such that

$$
\left(\int_{|x| \leq T}\left|\left(S_{d}^{*} f\right)(x)\right|^{p} d x\right)^{1 / p} \leq C\|f\|_{H_{s}}, \quad p \geq 1, s>0,
$$

for all $f \in \mathcal{S}\left(\mathbb{R}^{n}\right)$, then $\lim _{t \rightarrow 0+}(2 \pi)^{-n}\left(K_{t}^{d} * f\right)(x)=f(x)$ a.e. for all compactly supported $f \in H_{s}\left(\mathbb{R}^{n}\right)$. Hence the study of estimates of the form (1.4) and their various extensions is important. For example, Carbery [2] obtained

$$
\left(\int_{\mathbb{R}^{n}}\left|\left(S_{d}^{*} f\right)(x)\right|^{2} d x\right)^{1 / 2} \leq C\|f\|_{H_{s}}
$$

for $s>d / 2$, while it follows from a technique of Sjölin [9] that the weighted inequality

$$
\left(\int_{\mathbb{R}^{n}}\left|\left(S_{d}^{*} f\right)(x)\right|^{2} e^{-|x|^{2}} d x\right)^{1 / 2} \leq C\|f\|_{H_{s}}
$$

is satisfied for $d>1$ and $s>1 / 2$. The weighted estimate for the global maximal operator

$$
\left(\int_{\mathbb{R}^{n}}\left|\left(S_{d}^{* *} f\right)(x)\right|^{2} \frac{1}{(1+|x|)^{b}} d x\right)^{1 / 2} \leq C\|f\|_{H_{s}}
$$

with $b>1, s>b / 2, d>0$ was given by Vega [20], and the estimate

$$
\left(\int_{\mathbb{R}^{n}}\left|\left(S_{d}^{* *} f\right)(x)\right|^{2} \frac{d x}{|x|^{a}}\right)^{1 / 2} \leq C\|f\|_{H_{s}}
$$

which holds for $s>a / 2,0<d \leq a<n, a>1, n \geq 2$, and fails for $s<a / 2$, was proved by Heinig and Wang in [7]. Recently, Tao [18] has proved that (1.4) holds for $p=2, n=2$ and $s>2 / 5$. This is the best result obtained so far in dimensions greater than one.

The weight functions for the global maximal operator are essential, because (1.5) fails for $b=0$ and any $s \in \mathbb{R}([10])$. However, if $f$ is a radial function, more can be said. Sjölin [11] proved that (1.4) holds for all radial $f$ with $s=1 / 4$ if and only if $p \leq 4 n /(2 n-1)$, which is in contrast with a result of Wang [24], who showed that for non-radial $f$ and $p>2, d>1$, 
(1.4) fails with $s=1 / 4$. Note that (1.4) with $s=1 / 4, p=4$, and $n=1$ is sharp ([8]).

This leads to the following question: for what values of $p$ and $s$ is the global maximal estimate

$$
\left(\int_{\mathbb{R}^{n}}\left|\left(S_{d}^{* *} f\right)(x)\right|^{p} d x\right)^{1 / p} \leq C\|f\|_{H_{s}}
$$

satisfied for all radial functions $f$ ? This and related questions are discussed in this paper. In fact in Theorem 3.2, it is shown that (1.6) holds for $s>$ $n(1 / 2-1 / p)$ and fails for $s<n(1 / 2-1 / p)$, where $p \geq 2 n /(n-2), n \geq 3$ and $0<d \leq 2$. Moreover, it is shown in Theorem 3.3 that, for radial functions $f \in \mathcal{S}\left(\mathbb{R}^{n}\right), n \geq 3, n /(n-1)<d<n^{2} / 2(n-1)$, the estimate

$$
\left(\int_{\mathbb{R}^{n}}\left|\left(S_{d}^{* *} f\right)(x)\right|^{2 n /(n-d)} d x\right)^{(n-d) / 2 n} \leq C\|f\|_{H_{s}\left(\mathbb{R}^{n}\right)}
$$

holds for $s>d / 2$ and fails for $s<d / 2$. These results are proved using a general weighted inequality (Theorem 3.1), which in turn follows from a number of mixed-norm estimates involving general real-valued radial phase functions. The proofs of the mixed-norm estimates in this paper hinge on certain uniform pointwise estimates of the Bessel functions obtained by Guo [6], Stempak [16]-[17] and Vega [19]. These results, which may be of independent interest, are given in the next section together with some other preliminary estimates.

Throughout this paper, constants are denoted by $C$ (sometimes with subscripts) which may be different at different places. Inequalities given here are interpreted in the sense that if the right side is finite, so is the left side, and the inequality holds. For $0 \neq x \in \mathbb{R}^{n}$ we write $x^{\prime}=x /|x|$, where $x^{\prime} \in S^{n-1}$, the unit sphere in $\mathbb{R}^{n}$. The $L^{p}$-Sobolev spaces $H_{s}^{p}\left(\mathbb{R}^{n}\right), p \geq 1$, $s \in \mathbb{R}$, are defined by

$$
H_{s}^{p}\left(\mathbb{R}^{n}\right)=\left\{f \in \mathcal{S}^{\prime}\left(\mathbb{R}^{n}\right):\|f\|_{H_{s}^{p}}<\infty\right\},
$$

where

$$
\|f\|_{H_{s}^{p}}=\left(\int_{\mathbb{R}^{n}}\left|(1-\Delta)^{s / 2} f(x)\right|^{p} d x\right)^{1 / p}
$$

and $(1-\Delta)^{s / 2}$ is the Bessel potential operator given by

$$
(1-\Delta)^{s / 2} f(x)=(2 \pi)^{-n} \int_{\mathbb{R}^{n}} e^{i x \cdot \xi}\left(1+|\xi|^{2}\right)^{s / 2} \widehat{f}(\xi) d \xi, \quad f \in \mathcal{S}\left(\mathbb{R}^{n}\right) .
$$

The Riesz potential operator is defined by

$$
\left(I_{\alpha} f\right)(x)=C_{n} \int_{\mathbb{R}^{n}}|y-x|^{\alpha-n} f(y) d y, \quad f \in \mathcal{S}\left(\mathbb{R}^{n}\right), 0<\alpha<n,
$$


where $C_{n}$ is a constant chosen so that the Fourier transform (denoted by $\mathcal{F}$ ) of $I_{\alpha} f$ satisfies

$$
\mathcal{F}\left(I_{\alpha} f\right)(\xi)=|\xi|^{-\alpha} \widehat{f}(\xi) .
$$

For properties of these operators we refer to [14].

The Bessel function $J_{m}$ of order $m>-1 / 2$ is defined in integral form by

$$
J_{m}(r)=\frac{1}{\Gamma(1 / 2) \Gamma(m+1 / 2)}(r / 2)^{m} \int_{-1}^{1} e^{i r s}\left(1-s^{2}\right)^{(2 m-1) / 2} d s,
$$

where $r \geq 0$. The local and global maximal operators of $S_{P}$ are defined by

respectively

$$
\left(S_{P}^{*} f\right)(x)=\sup _{0<t<1}\left|\left(S_{P} f\right)(x, t)\right|,
$$

$$
\left(S_{P}^{* *} f\right)(x)=\sup _{t \in \mathbb{R}}\left|\left(S_{P} f\right)(x, t)\right| .
$$

Finally, the Fourier transform of $\left(S_{P} f\right)(x, t)$ in the $t$ variable is denoted by $\left(S_{P} f\right)^{\wedge}(x, \cdot)(\tau)$.

2. Mixed-norm estimates for $S_{P}^{* *}$. Weight functions are assumed to be non-negative locally integrable functions on $(0, \infty)$. Recall that the standard duality principle for $L^{p}$-spaces with weight $v$ is the following:

If $1<p<\infty, g(x) \geq 0, v(x) \geq 0, x \in(0, \infty)$, then

$$
\sup _{f \geq 0} \frac{\int_{0}^{\infty} f(x) g(x) d x}{\left(\int_{0}^{\infty} f(x)^{p} v(x) d x\right)^{1 / p}}=\left(\int_{0}^{\infty} g(x)^{q} v(x)^{1-q} d x\right)^{1 / q}
$$

here and throughout, the conjugate index $q$ of $p$ is defined by $1 / p+1 / q=1$.

The next lemma summarizes some known estimates of the Bessel functions $J_{m}(s)$.

LEMMA 2.1.

(i) If $v \geq 0$ is a non-negative real number, then

$$
\left|J_{v}(t)\right| \leq C
$$

where $C>0$ is independent of $v$ and $t>0$.

(ii) If $n \geq 2, v \geq 0$ and $p>4$, then

$$
\left(\int_{0}^{\infty} t\left|J_{v}(t)\right|^{p} d t\right)^{1 / p} \leq C
$$

where $C=C(p)>0$ is independent of $v \geq 0$.

(iii) If $n \geq 2, v \geq 0$ and $0<\gamma<n-1$, then

$$
\int_{0}^{\infty} t^{-\gamma}\left|J_{v}(t)\right|^{2} d t \leq C
$$

where $C=C(\gamma)>0$ is independent of $v$. 
Proof. (2.2) follows from [16; Eq. (4)] and [15; Lemma 3.11, Ch. 4] (see also [26; p. 176]). (2.3) follows from [6; Lemma 3.2] or [16; Eq. (3)] or [19; Lemma 2.2]. (2.4) follows from [26; p. 403]. More information on uniform pointwise and $L^{p}$ estimates of the Bessel functions $J_{v}(t)$ can be found in [16]-[17].

The following lemma is an immediate consequence of (2.19) and Theorem 3.10 of $[15 ;$ Ch.4].

Lemma 2.2. If $n \geq 2$ and $Y$ is a spherical harmonic of order $k$ on $S^{n-1}$, then

$$
\int_{S^{n-1}} e^{-i s\left(x^{\prime} \cdot \xi^{\prime}\right)} Y\left(\xi^{\prime}\right) d \sigma\left(\xi^{\prime}\right)=(2 \pi)^{n / 2} i^{-k} s^{(2-n) / 2} J_{(n+2 k-2) / 2}(s) Y\left(x^{\prime}\right) .
$$

Here $s>0, x^{\prime}, \xi^{\prime} \in S^{n-1}$ and $d \sigma$ denotes the surface measure on $S^{n-1}$.

From now on the phase function $P$ will be assumed to be radial and real-valued. The following lemma is instrumental in the proof of the main results of this paper. Note that a similar result was proved by Heinig and Wang [7; Lemma 2.4].

Lemma 2.3. Assume $n \geq 2, f \in \mathcal{S}\left(\mathbb{R}^{n}\right), w \geq 0$ is a radial weight function and $P(\xi)=\varphi(|\xi|), \xi \in \mathbb{R}^{n}$, where $\varphi \in C^{1}(0, \infty)$ is real-valued with $\left|\varphi^{\prime}(t)\right|>0$.

(i) If $1<p<\infty, 0<\gamma<n-1$, then for any $s_{0} \in \mathbb{R}$,

$$
\begin{aligned}
& \int_{\mathbb{R}^{n+1}}\left(1+\tau^{2}\right)^{s_{0}}\left|\left(S_{P} f\right)^{\wedge}(x, \cdot)(\tau)\right|^{2} w(|x|) d x d \tau \\
\leq & C\left(\int_{0}^{\infty} t^{q(1+\gamma / p)} w(t)^{q} d t\right)^{1 / q}\left(\int_{\mathbb{R}^{n}} \frac{\left(1+|\varphi(|\xi|)|^{2}\right)^{s_{0}}}{\left|\varphi^{\prime}(|\xi|)\right|}|\xi|^{1 / q+\gamma / p}|\widehat{f}(\xi)|^{2} d \xi\right),
\end{aligned}
$$

where $C>0$ depends only on $n, \gamma$, and $s_{0}$.

(ii) If $p>2$, then (2.6) holds with $\gamma=-1$ for all $s_{0} \in \mathbb{R}$, where $C>0$ depends on $n, p$, and $s_{0}$ only.

Proof. (i) We prove the case $\varphi^{\prime}(t)>0$; the proof for $\varphi^{\prime}(t)<0$ is identical. Let $A=\lim _{t \rightarrow 0+} \varphi(t), B=\lim _{t \rightarrow+\infty} \varphi(t)$ and $g \in \mathcal{S}(\mathbb{R})$. Then an interchange of the order of integration, a transformation to polar coordinates and a final change of variable yield

$$
\begin{aligned}
& \int_{\mathbb{R}}\left(S_{P} f\right)(x, t) \widehat{g}(t) d t=(2 \pi)^{-n} \int_{\mathbb{R}}\left(\int_{\mathbb{R}^{n}} e^{i x \cdot \xi} e^{i t P(\xi)} \widehat{f}(\xi) d \xi\right) \widehat{g}(t) d t \\
& =(2 \pi)^{-n} \int_{\mathbb{R}^{n}} e^{i x \cdot \xi} \widehat{f}(\xi) \int_{\mathbb{R}} e^{i t \varphi(|\xi|)} \widehat{g}(t) d t d \xi=(2 \pi)^{1-n} \int_{\mathbb{R}^{n}} e^{i x \cdot \xi} \widehat{f}(\xi) g(\varphi(|\xi|)) d \xi
\end{aligned}
$$




$$
\begin{aligned}
& =(2 \pi)^{1-n} \int_{0}^{\infty} r^{n-1} g(\varphi(r)) \int_{S^{n-1}} e^{i r|x|\left(x^{\prime} \cdot \xi^{\prime}\right)} \widehat{f}\left(r \xi^{\prime}\right) d \sigma\left(\xi^{\prime}\right) d r \\
& =(2 \pi)^{1-n} \int_{A}^{B} g(\tau) \frac{\left(\varphi^{-1}(\tau)\right)^{n-1}}{\varphi^{\prime}\left(\varphi^{-1}(\tau)\right)} \int_{S^{n-1}} e^{i \varphi^{-1}(\tau)|x|\left(x^{\prime} \cdot \xi^{\prime}\right)} \widehat{f}\left(\varphi^{-1}(\tau) \xi^{\prime}\right) d \sigma\left(\xi^{\prime}\right) d \tau,
\end{aligned}
$$

where the interchange of the order of integration is justified by Fubini's theorem. Hence $\left(S_{P} f\right)^{\wedge}(x, \cdot)(\tau)$ exists as a tempered distribution and is given by

$$
\begin{aligned}
& \left(S_{P} f\right)^{\wedge}(x, \cdot)(\tau) \\
& \quad=(2 \pi)^{1-n} \chi_{(A, B)}(\tau) \frac{\left(\varphi^{-1}(\tau)\right)^{n-1}}{\varphi^{\prime}\left(\varphi^{-1}(\tau)\right)} \int_{S^{n-1}} e^{i \varphi^{-1}(\tau)|x|\left(x^{\prime} \cdot \xi^{\prime}\right)} \widehat{f}\left(\varphi^{-1}(\tau) \xi^{\prime}\right) d \sigma\left(\xi^{\prime}\right)
\end{aligned}
$$

where $\chi_{(A, B)}$ is the characteristic function of the interval $(A, B)$.

Now, using polar coordinates, interchanging the order of integration and then making a change of variable yields

$$
\begin{aligned}
& \int_{\mathbb{R}^{n+1}}\left(1+\tau^{2}\right)^{s_{0}}\left|\left(S_{P} f\right)^{\wedge}(x, \cdot)(\tau)\right|^{2} w(|x|) d x d \tau \\
& =C \int_{\mathbb{R}^{n}} w(|x|) \int_{A}^{B}\left(1+\tau^{2}\right)^{s_{0}} \frac{\left|\varphi^{-1}(\tau)\right|^{2(n-1)}}{\left|\varphi^{\prime}\left(\varphi^{-1}(\tau)\right)\right|^{2}} \\
& \times\left|\int_{S^{n-1}} e^{i|x| \varphi^{-1}(\tau)\left(x^{\prime} \cdot \xi^{\prime}\right)} \widehat{f}\left(\varphi^{-1}(\tau) \xi^{\prime}\right) d \sigma\left(\xi^{\prime}\right)\right|^{2} d \tau d x \\
& \leq C \int_{0}^{\infty} r^{n-1} w(r) \int_{A}^{B}\left(1+\tau^{2}\right)^{s_{0}} \frac{\left|\varphi^{-1}(\tau)\right|^{2(n-1)}}{\left|\varphi^{\prime}\left(\varphi^{-1}(\tau)\right)\right|^{2}} \\
& \times \int_{S^{n-1}}\left|\int_{S^{n-1}} e^{i r \varphi^{-1}(\tau)\left(x^{\prime} \cdot \xi^{\prime}\right)} \widehat{f}\left(\varphi^{-1}(\tau) \xi^{\prime}\right) d \sigma\left(\xi^{\prime}\right)\right|^{2} d \tau d r d \sigma\left(x^{\prime}\right) \\
& \leq C \int_{0}^{\infty} r^{n-1} w(r) \int_{0}^{\infty}\left(1+|\varphi(t)|^{2}\right)^{s_{0}} \frac{t^{2(n-1)}}{\left|\varphi^{\prime}(t)\right|} \\
& \times \int_{S^{n-1}}\left|\int_{S^{n-1}} e^{i r t\left(x^{\prime} \cdot \xi^{\prime}\right)} \widehat{f}\left(t \xi^{\prime}\right) d \sigma\left(\xi^{\prime}\right)\right|^{2} d \sigma\left(x^{\prime}\right) d t d r \\
& \leq C \int_{0}^{\infty} \frac{\left(1+|\varphi(t)|^{2}\right)^{s_{0}}}{\left|\varphi^{\prime}(t)\right|} t^{2(n-1)} \int_{0}^{\infty} r^{n-1} w(r) \\
& \times \int_{S^{n-1}}\left|\int_{S^{n-1}} e^{i r t\left(x^{\prime} \cdot \xi^{\prime}\right)} \widehat{f}\left(t \xi^{\prime}\right) d \sigma\left(\xi^{\prime}\right)\right|^{2} d \sigma\left(x^{\prime}\right) d r d t .
\end{aligned}
$$


Let $H_{n}^{(k)}, n \geq 2$, be the finite-dimensional vector space of spherical harmonics $Y$ of order $k$ on $S^{n-1}$ with dimension say $d_{n, k}$ and an orthonormal basis $\left\{Y_{k, l}\right\}_{l=1}^{d_{n, k}}$. It follows from the theory of spherical harmonics as formulated in $\left[15\right.$; Ch. 4] that $\bigcup_{k=0}^{\infty}\left\{Y_{k, l}\right\}_{l=1}^{d_{n, k}}$ is a complete orthonormal basis of $L^{2}\left(S^{n-1}\right)$. If

$$
a_{k, l}(t)=\int_{S^{n-1}} \widehat{f}\left(t \xi^{\prime}\right) Y_{k, l}\left(\xi^{\prime}\right) d \sigma\left(\xi^{\prime}\right),
$$

then (2.5) and the orthogonality of $Y_{k, l}$ yield

$$
\begin{aligned}
\int_{S^{n-1}} \mid \int_{S^{n-1}} e^{i t r\left(x^{\prime} \cdot \xi^{\prime}\right)} \widehat{f}\left(t \xi^{\prime}\right) & \left.d \sigma\left(\xi^{\prime}\right)\right|^{2} d \sigma\left(x^{\prime}\right) \\
& =C \sum_{k=0}^{\infty} \sum_{l=1}^{d_{n, k}}\left|a_{k, l}(t)\right|^{2}(r t)^{2-n}\left|J_{(n+2 k-2) / 2}(r t)\right|^{2},
\end{aligned}
$$

and substituting this into (2.7) shows that

$$
\int_{\mathbb{R}^{n+1}}\left(1+\tau^{2}\right)^{s_{0}}\left|\left(S_{P} f\right)^{\wedge}(x, \cdot)(\tau)\right|^{2} w(|x|) d x d \tau
$$

$$
\leq C \int_{0}^{\infty} \frac{\left(1+|\varphi(t)|^{2}\right)^{s_{0}}}{\left|\varphi^{\prime}(t)\right|} t^{n}\left(\sum_{k=0}^{\infty} \sum_{l=1}^{d_{n, k}}\left|a_{k, l}(t)\right|^{2} \int_{0}^{\infty} r w(r)\left|J_{(n+2 k-2) / 2}(t r)\right|^{2} d r\right) d t .
$$

By Hölder's inequality and the estimates (2.2) and (2.4), we see that

$$
\begin{aligned}
& \int_{0}^{\infty} r w(r)\left|J_{(n+2 k-2) / 2}(t r)\right|^{2} d r \\
& =\int_{0}^{\infty} w(r) r^{(1+\gamma / p)} r^{-\gamma / p}\left|J_{(n+2 k-2) / 2}(t r)\right|^{2} d r \\
& \leq\left(\int_{0}^{\infty} r^{-\gamma}\left|J_{(n+2 k-2) / 2}(t r)\right|^{2 p} d r\right)^{1 / p}\left(\int_{0}^{\infty} w(r)^{q} r^{q(1+\gamma / p)} d r\right)^{1 / q} \\
& \leq C\left(\int_{0}^{\infty} r^{-\gamma}\left|J_{(n+2 k-2) / 2}(r t)\right|^{2} d r\right)^{1 / p}\left(\int_{0}^{\infty} w(r)^{q} r^{q(1+\gamma / p)} d r\right)^{1 / q} \\
& \leq C\left(t^{\gamma-1} \int_{0}^{\infty} u^{-\gamma}\left|J_{(n+2 k-2) / 2}(u)\right|^{2} d u\right)^{1 / p}\left(\int_{0}^{\infty} w(r)^{q} r^{q(1+\gamma / p)} d r\right)^{1 / q} \\
& \leq C t^{(\gamma-1) / p}\left(\int_{0}^{\infty} w(r)^{q} r^{q(1+\gamma / p)} d r\right)^{1 / q}
\end{aligned}
$$


where the constant $C>0$ is independent of $p, k \geq 0$ and $w$. Substituting this estimate into (2.8), we finally obtain

$$
\begin{aligned}
\int_{\mathbb{R}^{n+1}}(1+ & \left.\tau^{2}\right)^{s_{0}}\left|\left(S_{P} f\right)^{\wedge}(x, \cdot)(\tau)\right|^{2} w(|x|) d x d \tau \\
\leq & C\left(\int_{0}^{\infty} w(r)^{q} r^{(1+\gamma / p) q} d r\right)^{1 / q} \int_{0}^{\infty} \frac{\left(1+|\varphi(t)|^{2}\right)^{s_{0}}}{\left|\varphi^{\prime}(t)\right|} t^{n+(\gamma-1) / p} \\
& \times\left(\sum_{k=0}^{\infty} \sum_{l=1}^{d_{n, k}}\left|a_{k, l}(t)\right|^{2}\right) d t \\
= & C\left(\int_{0}^{\infty} w(r)^{q} r^{(1+\gamma / p) q} d r\right)^{1 / q} \int_{\mathbb{R}^{n}} \frac{\left(1+|\varphi(|\xi|)|^{2}\right)^{s_{0}}}{\left|\varphi^{\prime}(|\xi|)\right|}|\xi|^{1+(\gamma-1) / p}|\widehat{f}(\xi)|^{2} d \xi
\end{aligned}
$$

where $C>0$ depends only on $n, s_{0}$, and $\gamma$.

(ii) The proof of this part is very similar, the only difference lies in the estimation of the integral

$$
\int_{0}^{\infty} r w(r)\left|J_{(n+2 k-2) / 2}(r t)\right|^{2} d r .
$$

Since $2 p>4$, Hölder's inequality and the estimate (2.3) yield

$$
\begin{aligned}
\int_{0}^{\infty} w(r) r \mid & \left.J_{(n+2 k-2) / 2}(r t)\right|^{2} d r \\
& \leq\left(\int_{0}^{\infty} r\left|J_{(n+2 k-2) / 2}(r t)\right|^{2 p} d r\right)^{1 / p}\left(\int_{0}^{\infty} r w(r)^{q} d r\right)^{1 / q} \\
& \leq t^{-2 / p}\left(\int_{0}^{\infty} u\left|J_{(n+2 k-2) / 2}(u)\right|^{2 p} d u\right)^{1 / p}\left(\int_{0}^{\infty} r w(r)^{q} d r\right)^{1 / q} \\
& \leq C t^{-2 / p}\left(\int_{0}^{\infty} r w(r)^{q} d r\right)^{1 / q}
\end{aligned}
$$

where $C>0$ is independent of $k \geq 0$ and $w$. Proceeding as in the proof of (i), we deduce the result from (2.8) and (2.9).

Lemma 2.4. Let $n \geq 2, s_{0}>1 / 2, f \in \mathcal{S}\left(\mathbb{R}^{n}\right), w$ a non-negative measurable function defined on $(0, \infty)$, and $P(\xi)=\varphi(|\xi|)$, where $\varphi \in C^{1}(0, \infty)$ is real-valued with $\left|\varphi^{\prime}(t)\right|>0$.

(i) If $p>1,0<\gamma<n-1$, then there is a constant $C=C\left(n, \gamma, s_{0}\right)>0$ such that 


$$
\begin{aligned}
& \int_{\mathbb{R}^{n}}\left|\left(S_{P}^{* *} f\right)(x)\right|^{2} w(|x|) d x \\
\leq & C\left(\int_{0}^{\infty} t^{q(1+\gamma / p)} w(t)^{q} d t\right)^{1 / q}\left(\int_{\mathbb{R}^{n}} \frac{\left(1+|\varphi(|\xi|)|^{2}\right)^{s_{0}}}{\left|\varphi^{\prime}(|\xi|)\right|}|\xi|^{1 / q+\gamma / p}|\widehat{f}(\xi)|^{2} d \xi\right) .
\end{aligned}
$$

(ii) If $p>2$, then (2.10) holds with $\gamma=-1$ and $C=C\left(n, p, s_{0}\right)$.

Proof. Since

$$
\left(S_{P} f\right)(x, t)=(2 \pi)^{-1} \int_{\mathbb{R}} e^{i t \tau}\left(S_{P} f\right)^{\wedge}(x, \cdot)(\tau) d \tau,
$$

Schwarz's inequality yields

$$
\left(S_{P}^{* *} f\right)(x) \leq(2 \pi)^{-1}\left(\int_{\mathbb{R}}\left(1+\tau^{2}\right)^{-s_{0}} d \tau\right)^{1 / 2}\left(\int_{\mathbb{R}}\left(1+\tau^{2}\right)^{s_{0}}\left|\left(S_{P} f\right)^{\wedge}(x, \cdot)(\tau)\right|^{2} d \tau\right)^{1 / 2}
$$

and hence this lemma is an immediate consequence of Lemma 2.3.

We are now in a position to prove the following mixed-norm inequality for the global maximal function $\left(S_{P}^{* *} f\right)$ :

Theorem 2.1. Let $n \geq 2, s_{0}>1 / 2, f \in \mathcal{S}\left(\mathbb{R}^{n}\right)$, and $P(\xi)=\varphi(|\xi|)$, where $\varphi \in C^{1}(0, \infty)$ is real-valued with $\left|\varphi^{\prime}(t)\right|>0$.

(i) If $p \geq 2,0<\gamma<n-1$, then there exists a constant $C=C\left(n, \gamma, s_{0}\right)$ such that

$$
\begin{aligned}
& \left(\int_{0}^{\infty}\left(\int_{S^{n-1}}\left|\left(S_{P}^{* *} f\right)\left(r x^{\prime}\right)\right|^{2} d \sigma\left(x^{\prime}\right)\right)^{p / 2} r^{(n-2) p / 2-\gamma} d r\right)^{1 / p} \\
& \quad \leq C\left(\int_{\mathbb{R}^{n}} \frac{\left(1+|\varphi(|\xi|)|^{2}\right)^{s_{0}}}{\left|\varphi^{\prime}(|\xi|)\right|}|\xi|^{1+2(\gamma-1) / p}|\widehat{f}(\xi)|^{2} d \xi\right)^{1 / 2} .
\end{aligned}
$$

(ii) If $p>4$, then (2.11) holds with $\gamma=-1$ and $C=C\left(n, p, s_{0}\right)$.

Proof. (i) A transformation to polar coordinates in (2.10) yields

$$
\begin{array}{r}
\left(\int_{0}^{\infty} w(t) t^{n-1}\left(\int_{S^{n-1}}\left|\left(S_{P}^{* *} f\right)\left(t x^{\prime}\right)\right|^{2} d \sigma\left(x^{\prime}\right)\right) d t\right) /\left(\int_{0}^{\infty} w(t)^{q} t^{q(1+\gamma / p)} d t\right)^{1 / q} \\
\leq C\left(\int_{\mathbb{R}^{n}} \frac{\left(1+|\varphi(|\xi|)|^{2}\right)^{s_{0}}}{\left|\varphi^{\prime}(|\xi|)\right|}|\xi|^{1 / q+\gamma / p}|\widehat{f}(\xi)|^{2} d \xi\right) .
\end{array}
$$

Now using the duality principle (2.1) with $f$ equal to $w, v(t)=t^{q(1+\gamma / p)}$, $g$ defined by

$$
g(t)=t^{n-1}\left(\int_{S^{n-1}}\left|\left(S_{P}^{* *} f\right)\left(t x^{\prime}\right)\right|^{2} d \sigma\left(x^{\prime}\right)\right)
$$


and the roles of $p$ and $q$ interchanged, we obtain

$$
\begin{aligned}
\sup _{w \geq 0} \frac{\int_{0}^{\infty} w(t) t^{n-1}\left(\int_{S^{n-1}}\left|\left(S_{P}^{* *} f\right)\left(t x^{\prime}\right)\right|^{2} d \sigma\left(x^{\prime}\right)\right) d t}{} & \left(\int_{0}^{\infty} w(t)^{q} t^{q(1+\gamma / p)} d t\right)^{1 / q} \\
= & \left(\int_{0}^{\infty}\left(t^{n-1} \int_{S^{n-1}}\left|S_{P}^{* *} f\left(t x^{\prime}\right)\right|^{2} d \sigma\left(x^{\prime}\right)\right)^{p} t^{(1+\gamma / p) q(1-p)} d t\right)^{1 / p} \\
= & \left(\int_{0}^{\infty}\left[\int_{S^{n-1}}\left|S_{P}^{* *} f\left(t x^{\prime}\right)\right|^{2} d \sigma\left(x^{\prime}\right)\right]^{p} t^{p(n-2)-\gamma} d t\right)^{1 / p} \\
\leq & C \int_{\mathbb{R}^{n}} \frac{\left(1+|\varphi(|\xi|)|^{2}\right)^{s_{0}}}{\left|\varphi^{\prime}(|\xi|)\right|}|\xi|^{1 / q+\gamma / p}|\widehat{f}(\xi)|^{2} d \xi
\end{aligned}
$$

where $C=C\left(n, \gamma, s_{0}\right)$. The last inequality is equivalent to (2.11) by replacing $p$ by $p / 2$.

(ii) The proof is the same as that of (i), only now Lemma 2.4(ii) is applied.

If $f \in \mathcal{S}\left(\mathbb{R}^{n}\right)$ is radial, we immediately obtain

Corollary 2.1. Let $P(\xi)=\varphi(|\xi|), \xi \in \mathbb{R}^{n}, n \geq 2$, where $\varphi \in C^{1}(0, \infty)$ is real-valued with $\left|\varphi^{\prime}(t)\right|>0$ and $s_{0}>1 / 2$. Assume $f \in \mathcal{S}\left(\mathbb{R}^{n}\right)$ is radial.

(i) If $0<\gamma<n-1, p \geq 2$, then there exists a constant $C>0$, independent of $f$ and $p$, such that,

$$
\begin{aligned}
& \left(\int_{\mathbb{R}^{n}}\left|\left(S_{P}^{* *} f\right)(x)\right|^{p}|x|^{(n-2)(p-2) / 2-(1+\gamma)} d x\right)^{1 / p} \\
& \quad \leq C\left(\int_{\mathbb{R}^{n}} \frac{\left(1+|\varphi(|\xi|)|^{2}\right)^{s_{0}}}{\left|\varphi^{\prime}(|\xi|)\right|}|\xi|^{1+2(\gamma-1) / p}|\widehat{f}(\xi)|^{2} d \xi\right)^{1 / 2} .
\end{aligned}
$$

(ii) If $p>4$, then (2.12) holds with $\gamma=-1$.

(iii) The limiting case of (2.12) also holds

$$
\left.\sup _{x \in \mathbb{R}^{n}}\left|\left(S_{P}^{* *} f\right)(x)\right| x\right|^{(n-2) / 2} \mid \leq C\left(\int_{\mathbb{R}^{n}} \frac{\left(1+|\varphi(|\xi|)|^{2}\right)^{s_{0}}}{\left|\varphi^{\prime}(|\xi|)\right|}|\xi||\widehat{f}(\xi)|^{2} d \xi\right)^{1 / 2}
$$

Observe that (i) and (ii) follow from Theorem 2.1 since $f$ radial implies that $\widehat{f}$ and $S_{P}^{* *} f$ are radial. (iii) is obtained on taking the limit on both sides of the inequality (2.12) as $p \rightarrow \infty$ and using the fact that the constant $C>0$ in (2.11) is independent of $p$.

Since

$$
S_{P}^{* *}\left(\mathcal{F}^{-1} f\right)(x) \geq(2 \pi)^{n}|\widehat{f}(-x)|,
$$


(2.11) with $\varphi(t)=t$ and $f$ replaced by $\widehat{f}$ yields the Fourier inequality for radial functions,

$$
\begin{aligned}
\left(\int_{\mathbb{R}^{n}}|\widehat{f}(x)|^{p}|x|^{(n-2)(p-2) / 2-(1+\gamma)} d x\right)^{1 / p} & \\
& \leq C\left(\int_{\mathbb{R}^{n}}\left(1+|\xi|^{2}\right)^{s_{0}}|\xi|^{1+2(\gamma-1) / p}|f(\xi)|^{2} d \xi\right)^{1 / 2} .
\end{aligned}
$$

This result is of some interest since the weight function $|x|^{(n-2)(p-2) / 2-(1+\gamma)}$ may be increasing.

3. Integral estimates of $S_{d}^{* *}$ for radial $f$. The main results for $S_{d}^{* *}, d>0$, valid for radial functions, are given next.

THEOREM 3.1. Let $n \geq 2$.

(i) If $0<\gamma<n-1, p \geq 2$, and $0<d / 2 \leq 1+(\gamma-1) / p$, then

$$
\left(\int_{\mathbb{R}^{n}}\left|\left(S_{d}^{* *} f\right)(x)\right|^{p}|x|^{(n-2)(p-2) / 2-(1+\gamma)} d x\right)^{1 / p} \leq C\|f\|_{H_{s}}
$$

holds for all radial functions $f \in \mathcal{S}\left(\mathbb{R}^{n}\right)$ if $s>1+(\gamma-1) / p$, and fails for $s<1+(\gamma-1) / p$.

(ii) If $p>4$ and $0<d<n$, then

$$
\left(\int_{\mathbb{R}^{n}}\left|\left(S_{d}^{* *} f\right)(x)\right|^{p}|x|^{(n-2)(p-2) / 2} d x\right)^{1 / p} \leq C\|f\|_{H_{s}^{r}}
$$

for all radial $f \in \mathcal{S}\left(\mathbb{R}^{n}\right)$, all $s>d / 2+(1-2 / p)$ and $r=2 n /(n+d)$.

Proof. (i) Choose $s_{0}=1 / 2+\varepsilon$ and $\varphi(t)=t^{d}$ in Corollary 2.1(i). Since $2+2(\gamma-1) / p \geq d$, it follows that

$$
\begin{aligned}
\left(\int_{\mathbb{R}^{n}}\left|\left(S_{d}^{* *} f\right)(x)\right|^{p}|x|^{(n-2)(p-2) / 2-(1+\gamma)} d x\right)^{1 / p} & \\
& \leq C\left(\int_{\mathbb{R}^{n}}\left(1+|\xi|^{2}\right)^{(1 / 2+\varepsilon) d}|\xi|^{2+2(\gamma-1) / p-d}|\widehat{f}(\xi)|^{2}\right)^{1 / 2} \\
& \leq C\|f\|_{H_{(1 / 2+\varepsilon) d+1+(\gamma-1) / p-d / 2}} \leq C\|f\|_{H_{1+(\gamma-1) / p+d \varepsilon}}
\end{aligned}
$$

from which the positive result of (i) follows. To complete the proof of (i), we require a lemma on weighted Fourier inequalities for radial functions.

Lemma 3.1. Assume $n \geq 1, a, s_{0}$ are real numbers and $p \geq 2$. If

$$
\left(\int_{\mathbb{R}^{n}}|\widehat{f}(x)|^{p} \frac{d x}{|x|^{a}}\right)^{1 / p} \leq C\left(\int_{\mathbb{R}^{n}}|f(x)|^{2}\left(1+|x|^{2}\right)^{s_{0}} d x\right)^{1 / 2}
$$


holds for all radial functions $f \in \mathcal{S}\left(\mathbb{R}^{n}\right)$, then $s_{0} \geq n(1 / 2-1 / p)+a / p$ and $a<n$.

Proof. Let $f$ be radial, so $f(x)=g(t), t=|x|$. Then

$$
\left(\int_{\mathbb{R}^{n}}\left(1+|x|^{2}\right)^{s_{0}}|f(x)|^{2} d x\right)^{1 / 2}=C\left(\int_{0}^{\infty}\left|\left(1+r^{2}\right)^{s_{0} / 2} r^{(n-1) / 2} g(r)\right|^{2} d r\right)^{1 / 2} .
$$

Moreover since $f$ is radial, so is $\widehat{f}$, and by [5],

$$
\begin{aligned}
\widehat{f}(\xi) & =(2 \pi)^{n / 2}|\xi|^{1-n / 2} \int_{0}^{\infty} r^{n / 2} g(r) J_{(n / 2-1)}(r|\xi|) d r \\
& =(2 \pi)^{n / 2}|\xi|^{(1-n) / 2}\left(H_{n / 2-1} F\right)(|\xi|),
\end{aligned}
$$

where $F(r)=r^{(n-1) / 2} g(r)$ and $\left(H_{n / 2-1} F\right)(s)=\int_{0}^{\infty}(r s)^{1 / 2} F(r) J_{n / 2-1}(r s) d r$ is the Hankel transform of $F$ of order $n / 2-1$. Therefore, using polar coordinates we get

$$
\begin{aligned}
\left(\int_{\mathbb{R}^{n}}|\widehat{f}(x)|^{p} \frac{d x}{|x|^{a}}\right)^{1 / p} & =C\left(\int_{0}^{\infty} s^{n-1} s^{-a}\left|s^{(1-n) / 2}\left(H_{n / 2-1} F\right)(s)\right|^{p} d s\right)^{1 / p} \\
& =C\left(\int_{0}^{\infty}\left|s^{(n-1)(1 / p-1 / 2)-a / p}\left(H_{n / 2-1} F\right)(s)\right|^{p} d s\right)^{1 / p}
\end{aligned}
$$

and hence inequality (3.3) has the form

$$
\left(\int_{0}^{\infty}\left|U(s)\left(H_{n / 2-1} F\right)(s)\right|^{p} d s\right)^{1 / p} \leq C\left(\int_{0}^{\infty}|V(s) F(s)|^{2} d s\right)^{1 / 2}
$$

where $F(r)=r^{(n-1) / 2} g(r), U(s)=s^{(1 / p-1 / 2)(n-1)-a / p}$ and $V(s)=\left(1+s^{2}\right)^{s_{0} / 2}$.

By [5; Thm. 3, Remark 1], this estimate implies

$$
\sup _{s>0}\left(\int_{0}^{1 / s} U(t)^{p} t^{(n-1) p / 2} d t\right)^{1 / p}\left(\int_{0}^{s} t^{n-1} V(t)^{-2} d t\right)^{1 / 2}<\infty,
$$

or equivalently

$$
\sup _{s>0}\left[\int_{0}^{1 / s} t^{n-1-a} d t\right]^{1 / p}\left[\int_{0}^{s}\left(1+t^{2}\right)^{-s_{0}} t^{n-1} d t\right]^{1 / 2}<\infty .
$$

Since the integral on the left will not be finite if $a \geq n$, it follows that $a<n$.

If $s>0$ is large, then

$$
\left[\int_{0}^{1 / s} t^{n-1-a} d t\right]^{1 / p} \geq C(1 / s)^{(n-a) / p}
$$


and

$$
\left[\int_{0}^{s}\left(1+t^{2}\right)^{-s_{0}} t^{n-1} d t\right]^{1 / 2} \geq C\left[\int_{0}^{s} t^{-2 s_{0}+n-1} d t\right]^{1 / 2} \geq C s^{\left(n-2 s_{0}\right) / 2} .
$$

Substituting these estimates into (3.4) yields

$$
\begin{aligned}
\infty & >\sup _{s>0}\left[\int_{0}^{1 / s} t^{n-1-a} d t\right]^{1 / p}\left[\int_{0}^{s}\left(1+t^{2}\right)^{-s_{0}} t^{n-1} d t\right]^{1 / 2} \\
& \geq \limsup _{s \rightarrow \infty}\left[\int_{0}^{1 / s} t^{n-1-a} d t\right]^{1 / p}\left[\int_{0}^{s}\left(1+t^{2}\right)^{-s_{0}} t^{n-1} d t\right]^{1 / 2} \\
& \geq \limsup _{s \rightarrow \infty} C(1 / s)^{(n-a) / p} s^{\left(n-2 s_{0}\right) / 2} .
\end{aligned}
$$

Hence $-(n-a) / p+\left(n-2 s_{0}\right) / 2 \leq 0$. This proves the lemma.

Now we complete the proof of Theorem 3.1(i).

If (3.1) holds for all radial $f \in \mathcal{S}\left(\mathbb{R}^{n}\right)$, then in particular it holds with $f$ replaced by $\mathcal{F}^{-1} f$, where $\mathcal{F}^{-1}$ denotes the inverse Fourier transform. But since $S_{d}^{* *}\left(\mathcal{F}^{-1} f\right)(x) \geq(2 \pi)^{n}|\widehat{f}(-x)|$, one obtains

$$
\left(\int_{\mathbb{R}^{n}}|\widehat{f}(x)|^{p}|x|^{(n-2)(p-2) / 2-(1+\gamma)} d x\right)^{1 / p} \leq C\left(\int_{\mathbb{R}^{n}}\left(1+|x|^{2}\right)^{s}|f(x)|^{2} d x\right)^{1 / 2} .
$$

Hence by Lemma 3.1 with $s_{0}=s$ and

$$
a=-[(n-2)(p-2) / 2-(1+\gamma)],
$$

it follows that

$$
\begin{aligned}
s & \geq n(1 / 2-1 / p)+a / p \\
& =n(1 / 2-1 / p)-(n-2)(1 / 2-1 / p)+(1+\gamma) / p \\
& =n(1 / 2-1 / p)-n(1 / 2-1 / p)+2(1 / 2-1 / p)+(1+\gamma) / p \\
& =1+(\gamma-1) / p .
\end{aligned}
$$

Therefore, if $s<1+(\gamma-1) / p$, then (3.1) fails for radial functions.

To prove (ii), choose $s_{0}=1 / 2+\varepsilon$ and $\varphi(t)=t^{d}$ in Corollary 2.1(ii). Then by (2.11) with $\gamma=-1$,

$$
\begin{aligned}
& \left(\int_{\mathbb{R}^{n}}\left|\left(S_{d}^{* *} f\right)(x)\right|^{p}|x|^{(n-2)(p-2) / 2} d x\right)^{1 / p} \\
& \quad \leq C\left(\int_{\mathbb{R}^{n}}\left(1+|\xi|^{2}\right)^{(1 / 2+\varepsilon) d}|\xi|^{2-4 / p-d}|\widehat{f}(\xi)|^{2} d \xi\right)^{1 / 2}
\end{aligned}
$$




$$
\begin{aligned}
& \leq C\left(\int_{\mathbb{R}^{n}}\left(1+|\xi|^{2}\right)^{(1 / 2+\varepsilon) d+1-2 / p}|\xi|^{-d}|\widehat{f}(\xi)|^{2} d \xi\right)^{1 / 2} \\
& =C\left(\int_{\mathbb{R}^{n}}\left|\mathcal{F}\left(I_{d / 2}(1-\Delta)^{[(1 / 2+\varepsilon) d+1-2 / p] / 2} f\right)(x)\right|^{2} d x\right)^{1 / 2} \\
& =C\left(\int_{\mathbb{R}^{n}}\left|\left(I_{d / 2}(1-\Delta)^{[(1 / 2+\varepsilon) d+1-2 / p] / 2} f\right)(x)\right|^{2} d x\right)^{1 / 2} \\
& \leq C\left(\int_{\mathbb{R}^{n}}\left|(1-\Delta)^{[(1 / 2+\varepsilon) d+1-2 / p] / 2} f(x)\right|^{r} d x\right)^{1 / r} \\
& =C\|f\|_{H_{(1 / 2+\varepsilon) d+1-2 / p}^{r}}
\end{aligned}
$$

where $\mathcal{F}, I_{d / 2}$, and $(1-\Delta)^{[(1 / 2+\varepsilon) d+1-2 / p] / 2}$ denote the Fourier transform, the Riesz potential operator of order $d / 2$ and the Bessel potential operator of order $-[(1 / 2+\varepsilon) d+1-2 / p]$, respectively. Here, Plancherel's theorem and the Hardy-Littlewood-Sobolev inequality were applied with

$$
1-\frac{d}{2 n}=1-\left(\frac{1}{r}-\frac{1}{2}\right)
$$

that is, $r=2 n /(n+d)$ (cf. [14]). This completes the proof of Theorem 3.1.

An interpolation argument and Theorem 3.1(i) yield the following sharp non-weighted result:

TheOrem 3.2. Assume $n \geq 3,0<d \leq 2$, and $p \geq 2 n /(n-2)$. Then

$$
\left(\int_{\mathbb{R}^{n}}\left|\left(S_{d}^{* *} f\right)(x)\right|^{p} d x\right)^{1 / p} \leq C\|f\|_{H_{s}}
$$

holds for all radial $f \in \mathcal{S}\left(\mathbb{R}^{n}\right)$ if $s>n(1 / 2-1 / p)$, and fails for $s<$ $n(1 / 2-1 / p)$.

Proof. Let $\gamma=1, s_{1}=1+\varepsilon, \varepsilon>0$ and choose $p=p_{1}=2 n /(n-2)$ in Theorem 3.1(i). Then (3.1) takes the form

$$
\left(\int_{\mathbb{R}^{n}}\left|\left(S_{d}^{* *} f\right)(x)\right|^{p_{1}} d x\right)^{1 / p_{1}} \leq C\|f\|_{H_{s_{1}}} .
$$

Also, by Schwarz's inequality,

$$
\begin{aligned}
\left\|S_{d}^{* *} f\right\|_{\infty} & =\sup _{x \in \mathbb{R}^{n}}\left|\left(S_{d}^{* *} f\right)(x)\right| \leq(2 \pi)^{-n} \int_{\mathbb{R}^{n}}|\widehat{f}(\xi)| d \xi \\
& \leq(2 \pi)^{-n}\left(\int_{\mathbb{R}^{n}}\left(1+|\xi|^{2}\right)^{-n / 2-\varepsilon} d \xi\right)^{1 / 2}\left(\int_{\mathbb{R}^{n}}\left(1+|\xi|^{2}\right)^{n / 2+\varepsilon}|\widehat{f}(\xi)|^{2} d \xi\right)^{1 / 2} \\
& \leq C\|f\|_{H_{n / 2+\varepsilon}}
\end{aligned}
$$


Interpolating between this and (3.6) yields

$$
\left(\int_{\mathbb{R}^{n}}\left|\left(S_{d}^{* *} f\right)(x)\right|^{p} d x\right)^{1 / p} \leq C\|f\|_{H_{s}}
$$

where $1 / p=\theta / p_{1}, 0<\theta<1$, and

$$
\begin{aligned}
s & =s_{1} \theta+(n / 2+\varepsilon)(1-\theta)=(1+\varepsilon) \theta+(n / 2+\varepsilon)(1-\theta) \\
& =\theta+(n / 2)(1-\theta)+\varepsilon=p_{1} / p+(n / 2)-(n / 2) p_{1} / p+\varepsilon \\
& =((1-n / 2) / p)(2 n /(n-2))+n / 2+\varepsilon=n(1 / 2-1 / p)+\varepsilon .
\end{aligned}
$$

Hence (3.5) holds for $s>n(1 / 2-1 / p)$.

The fact that $(3.5)$ fails if $s<n(1 / 2-1 / p)$ is a direct consequence of Lemma 3.1.

Theorem 3.3. Assume $n \geq 3$ and

$$
\frac{n}{n-1}<d<\frac{n^{2}}{2(n-1)}
$$

Then

$$
\left(\int_{\mathbb{R}^{n}}\left|\left(S_{d}^{* *} f\right)(x)\right|^{2 n /(n-d)} d x\right)^{(n-d) / 2 n} \leq C\|f\|_{H_{s}}
$$

holds for all radial $f \in \mathcal{S}\left(\mathbb{R}^{n}\right)$ if $s>d / 2$, and fails for $s<d / 2$.

Proof. In (3.1), by setting $a=1+\gamma$ and $(n-2)(p-2) / 2-(1+\gamma)=0$, one obtains $1<a<n, p=2+2 a /(n-2), 1+(\gamma-1) / p=n(1 / 2-1 / p)=$ $n a / 2(n-2+a), 0<d \leq 2(1+(\gamma-1) / p)=n a /(n-2+a)$ and

$$
\left(\int_{\mathbb{R}^{n}}\left|\left(S_{d}^{* *} f\right)(x)\right|^{p} d x\right)^{1 / p} \leq C\|f\|_{H_{s}}, \quad n \geq 3,
$$

holds for all radial functions $f \in \mathcal{S}\left(\mathbb{R}^{n}\right)$ if $s>n(1 / 2-1 / p)$, and fails for $s<n(1 / 2-1 / p)$. Since

$$
\frac{d}{d a}\left(\frac{n a}{n-2+a}\right)=\frac{n(n-2)}{(n-2+a)^{2}}>0, \quad a>0, n \geq 3,
$$

it follows that

$$
\frac{n}{n-1}<\frac{n a}{n-2+a}<\frac{n^{2}}{2(n-1)}<n, \quad 1<a<n, n \geq 3 .
$$

Hence for any $n \geq 3$ and $n /(n-1)<d<n^{2} / 2(n-1)$, the equation $d=$ $n a /(n-2+a)$ has a unique solution $a$ in the interval $1<a<n$. In fact, one obtains

$$
a=\frac{d(n-2)}{n-d}, \quad p=2+\frac{2 a}{n-2}=\frac{2 n}{n-d}
$$

and

$$
n(1 / 2-1 / p)=n(1 / 2-(n-d) / 2 n)=d / 2 .
$$


It follows that for $n \geq 3, n /(n-1)<d<n^{2} / 2(n-1)$ and $p=2 n /(n-d)$, (3.8) holds for $s>d / 2$ and fails for $s<d / 2$. This completes the proof of Theorem 3.3.

An interpolation argument almost identical to that in the proof of Theorem 3.2 shows that for $n \geq 3, n /(n-1)<d<n^{2} / 2(n-1)$ and $p \geq$ $2 n /(n-d),(3.8)$ holds for $s>n(1 / 2-1 / p)$ and fails for $s<n(1 / 2-1 / p)$.

Acknowledgments. The author thanks the anonymous reviewer for providing comments and corrections that greatly improved this paper. He would like to thank his $\mathrm{PhD}$ advisor, Professor Hans P. Heinig, for encouragement and friendship over the years. He would also like to thank Professors K. Guo, P. Sjölin, L. Vega and B. G. Walther for providing papers and preprints.

\section{References}

[1] J. Bergh and J. Löfström, Interpolation Spaces. An Introduction, Springer, 1976.

[2] A. Carbery, Radial Fourier multipliers and associated maximal functions, in: NorthHolland Math. Stud. 111, 1985, 49-55.

[3] L. Carleson, Some analytical problems related to statistical mechanics, in: Euclidean Harmonic Analysis, Lecture Notes in Math. 779, Springer, 1979, 5-45.

[4] B. E. J. Dahlberg and C. E. Kenig, A note on the almost everywhere behavior of solutions to the Schrödinger equation, in: Harmonic Analysis, Lecture Notes in Math. 908, Springer, 1982, 205-209.

[5] S. A. Emara and H. P. Heinig, Weighted norm inequalities for the Hankel- and K-transformations, Proc. Roy. Soc. Edinburgh Sect. A 103 (1986), 325-333.

[6] K. Guo, A uniform $L^{p}$-estimate of Bessel functions and distributions supported on $S^{n-1}$, Proc. Amer. Math. Soc. 125 (1997), 1329-1340.

[7] H. P. Heinig and S. Wang, Maximal function estimates of solutions to general dispersive partial differential equations, Trans. Amer. Math. Soc. 351 (1999), 79-108.

[8] C. E. Kenig, G. Ponce and L. Vega, Oscillatory integrals and regularity of dispersive equations, Indiana Univ. Math. J. 40 (1991), 33-69.

[9] P. Sjölin, Regularity of solutions to the Schrödinger equation, Duke Math. J. 55 (1987), 699-715.

[10] - Global maximal estimates for solutions to the Schrödinger equation, Studia Math. 110 (1994), 105-114.

[11] —, Radial functions and maximal estimates for solutions to the Schrödinger equation, J. Austral. Math. Soc. Ser. A 59 (1995), 134-142.

[12] - $L^{p}$ maximal estimates for solutions to the Schrödinger equation, Math. Scand. 81 (1997), 35-68.

[13] - , A counter-example concerning maximal estimates for solutions to equations of Schrödinger type, Indiana Univ. Math. J. 47 (1998), 593-599.

[14] E. M. Stein, Singular Integrals and Differentiability Properties of Functions, Princeton Univ. Press, 1970.

[15] E. M. Stein and G. Weiss, Introduction to Fourier Analysis on Euclidean Spaces, Princeton Univ. Press, 1971. 
[16] K. Stempak, A weighted uniform $L^{p}$-estimate of Bessel functions: a note on a paper of Guo, Proc. Amer. Math. Soc. 128 (2000), 2943-2945.

[17] —, Uniform two-weight norm inequalities for Hankel transform partial sum operators, Houston J. Math. 29 (2003), 1045-1063.

[18] T. Tao, A sharp bilinear restriction estimate for paraboloids, Geom. Funct. Anal. 13 (2002), 1359-1384.

[19] L. Vega, El multiplicador de Schrödinger, la función maximal y los operadores de restricción, Doctoral Thesis, Dept. Mat., Univ. Autónoma de Madrid, 1988.

[20] —, Schrödinger equations: pointwise convergence to the initial data, Proc. Amer. Math. Soc. 102 (1988), 874-878.

[21] B. G. Walther, Maximal estimates of oscillatory integrals with concave phase, in: Harmonic Analysis and Operator Theory, Contemp. Math. 189, Amer. Math. Soc., $1995,485-495$.

[22] - Homogeneous estimates for oscillatory integrals, Acta Math. Univ. Comenian. 9 (2000), 151-171.

[23] - , Higher integrability for maximal oscillatory Fourier integrals, Ann. Acad. Sci. Fenn. Math. 26 (2001), 189-204.

[24] S. Wang, On the maximal operator associated with the free Schrödinger equation, Studia Math. 122 (1997), 167-182.

[25] —, Maximal estimates of solutions to dispersive partial differential equations, Doctoral Thesis, Dept. Math. \& Stats., McMaster Univ., 1996.

[26] G. N. Watson, A Treatise on the Theory of Bessel Functions, Cambridge Univ. Press, 1922.

Defence Research and Development Canada-Ottawa

3701 Carling Avenue

Ottawa, Ontario, Canada K1A 0Z4

E-mail: sichun.wang@drdc-rddc.gc.ca

Received May 31, 2005

Revised version April 6, 2006 